\title{
17ß-estradiol or triclosan-induced epithelial-mesenchymal transition and migration of MCF-7 breast cancer cells were reversed by kaempferol, a phytoestrogen
}

Geuma Lee, Kyung-A Hwang and Kyung-Chul Choi

Laboratory of Veterinary Biochemistry and Immunology, College of Veterinary Medicine, Chungbuk National University, Cheongju, Chungbuk, Republic of Korea

\section{ABSTRACT}

Triclosan (TCS) is an endocrine-disrupting chemical (EDC) and has a potential to increase progression of hormone responsive cancers as does 17ß-estradiol (E2). As a phytoestrogen, Kaempferol plays a chemopreventive role inhibiting cancer formation and progression. In this study, chemopreventive activity of kaempferol was examined by measuring its effect on growth, epithelialmesenchymal transition (EMT), and migration of MCF-7 breast cancer cells increased by 17ß-estradiol (E2, a positive control) or triclosan (TCS), an endocrinedisrupting chemical (EDC). As an EDC, TCS is known to interfere estrogen receptor (ER) dependent pathway in MCF-7 cells expressing ERs. In MTT assay, TCS $\left(1^{-4}-10^{-7}\right.$ M) or E2 $\left(10^{-9} \mathrm{M}\right)$ induced cell growth of MCF-7 cells, which was reversed to a control level by co-treatment of ICI 182,780 (10-8 M), an ER antagonist, or kaempferol (50 $\mathrm{uM})$. In a wound-healing scratch assay, TCS enhanced migration of MCF-7 cells like E2, but co-treatment of kaempferol or ICI 182,720 decreased the migration ability of MCF-7 cells to a control level. Also Kaempferol could block invasion ability of MCF-7 breast cancer cell induced by $\mathrm{E} 2$ and TCS in transwell invasion assay. In western blot assay, we examined the effect of TCS and Kaempferol on protein expression of EMT-related markers such as Ecadherin, N-cadherin, snail and slug. TCS induced the increased expression of EMT promoting markers such as N-cadherin, snail and slug, but down-regulated the expression of E-cadherin, an epithelial marker inhibiting EMT process. On the contrary, kaempferol was shown to reverse the expression pattern of EMT-related markers induced by TCS or E2. Also, E2 and TCS induced metastasis in MCF-7 breast cancer cell. We examined the effect of E2, TCS and Kaempferol on protein expression of metastasis related markers such as Cathepsin $B$ and $D$. E2 and TCS increased the expression of Cathepsin B and D, but ICI 182,780 and Kaempferol decreased the expression of cathepsin $B$ and $D$. In conclusion, kaempferol effectively suppressed growth, EMT, and migration ability of MCF-7 breast cancer cells increased by E2 and TCS.

\section{RESULTS}

(A)

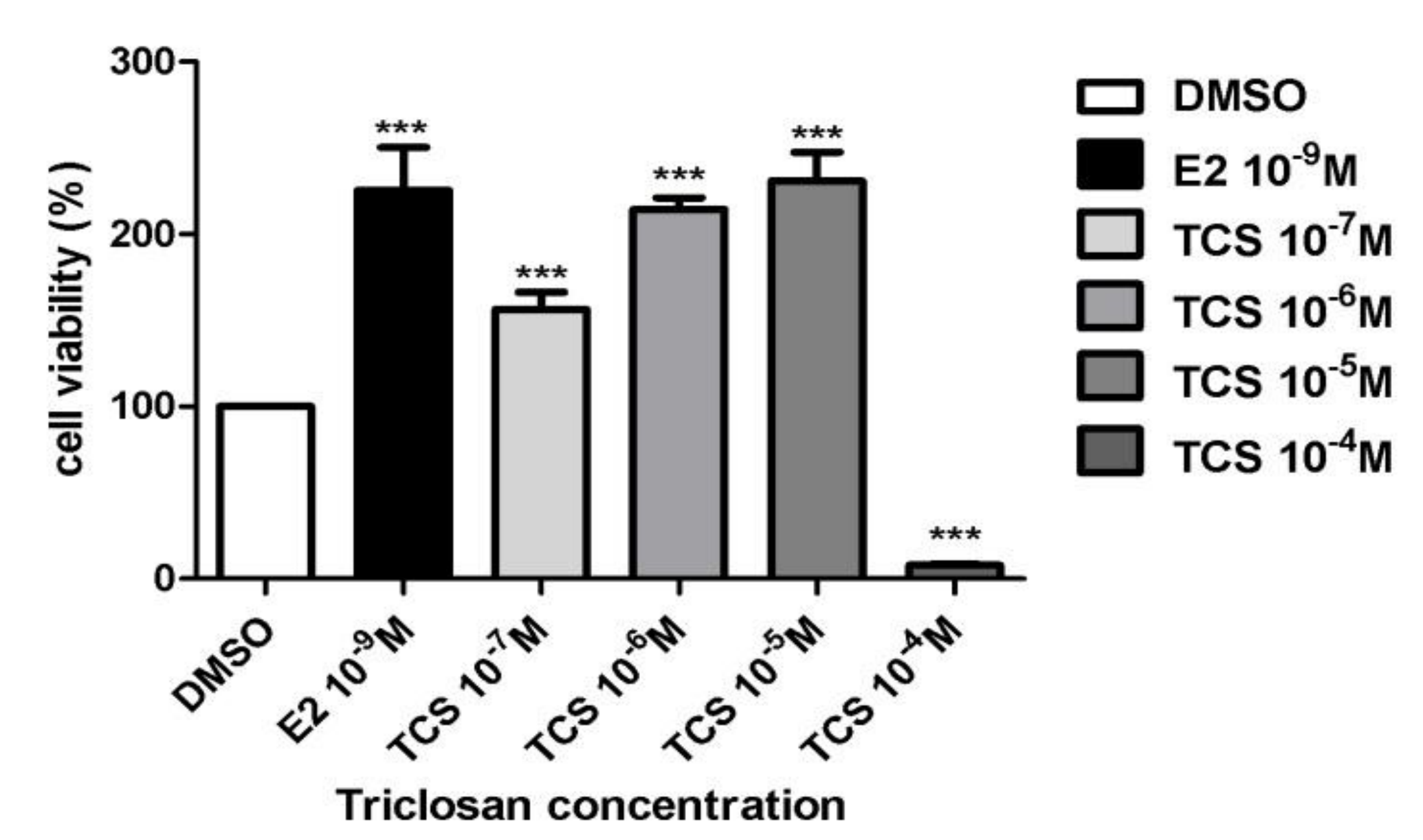

(B)

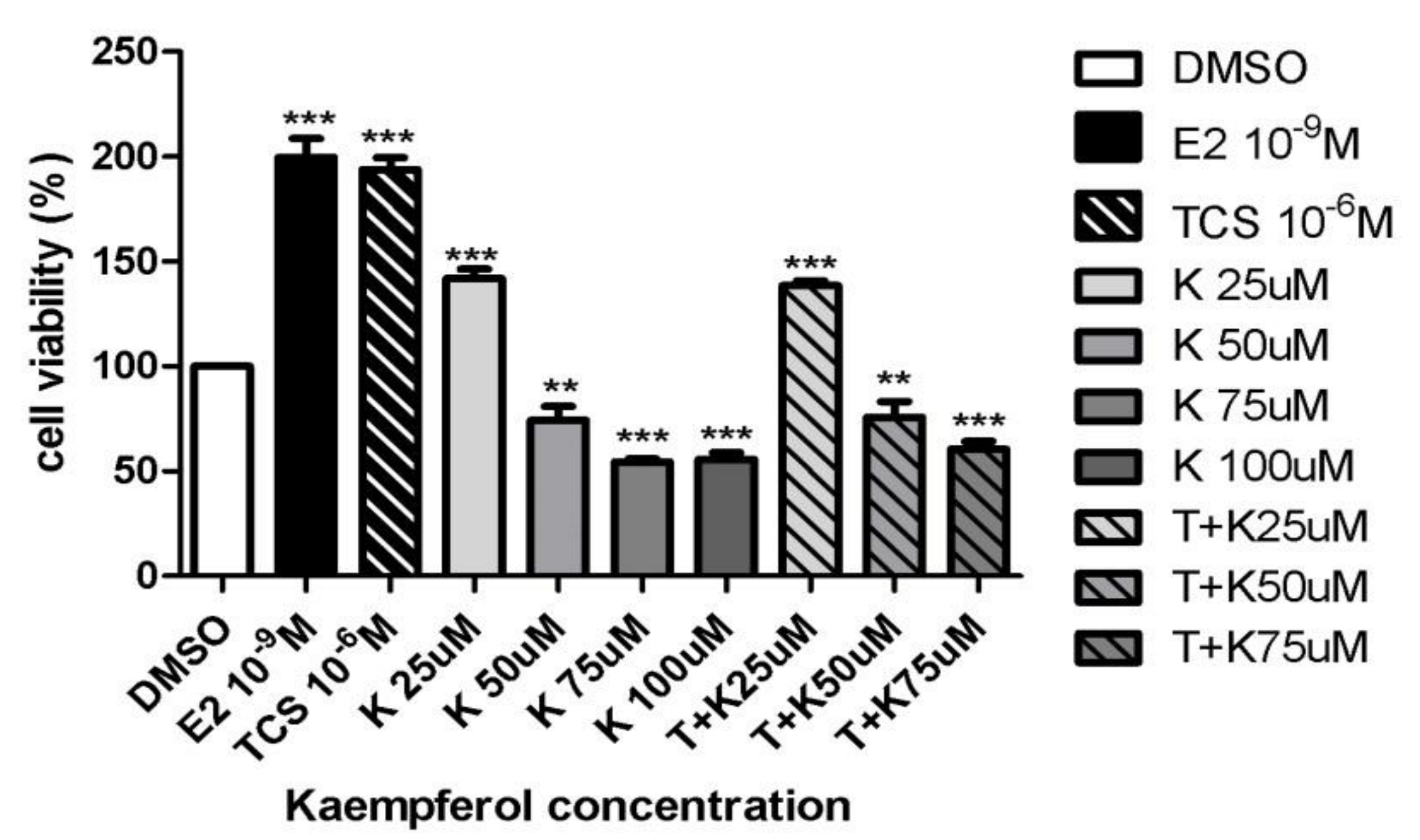

Figure 1. Effects of E2, TCS or Kaem on cell proliferation of MCF-7 cells. MCF-7 cells were seeded at 4,000 cells/well in 96well plates in the presence of phenol-free DMEM $(+5 \% \mathrm{CD}$ FBS). After 2 days, the medium was replaced by phenol red-free DMEM (+5\% CD-FBS) containing TCS (A) and Kaem and Kaem+TCS (B) at the concentrations indicated for 6 days. The cell viability was determined by MTT assay. * $\mathrm{P}<0.05$ compare to a vehicle treated with DMSO.

RESULTS

(A)

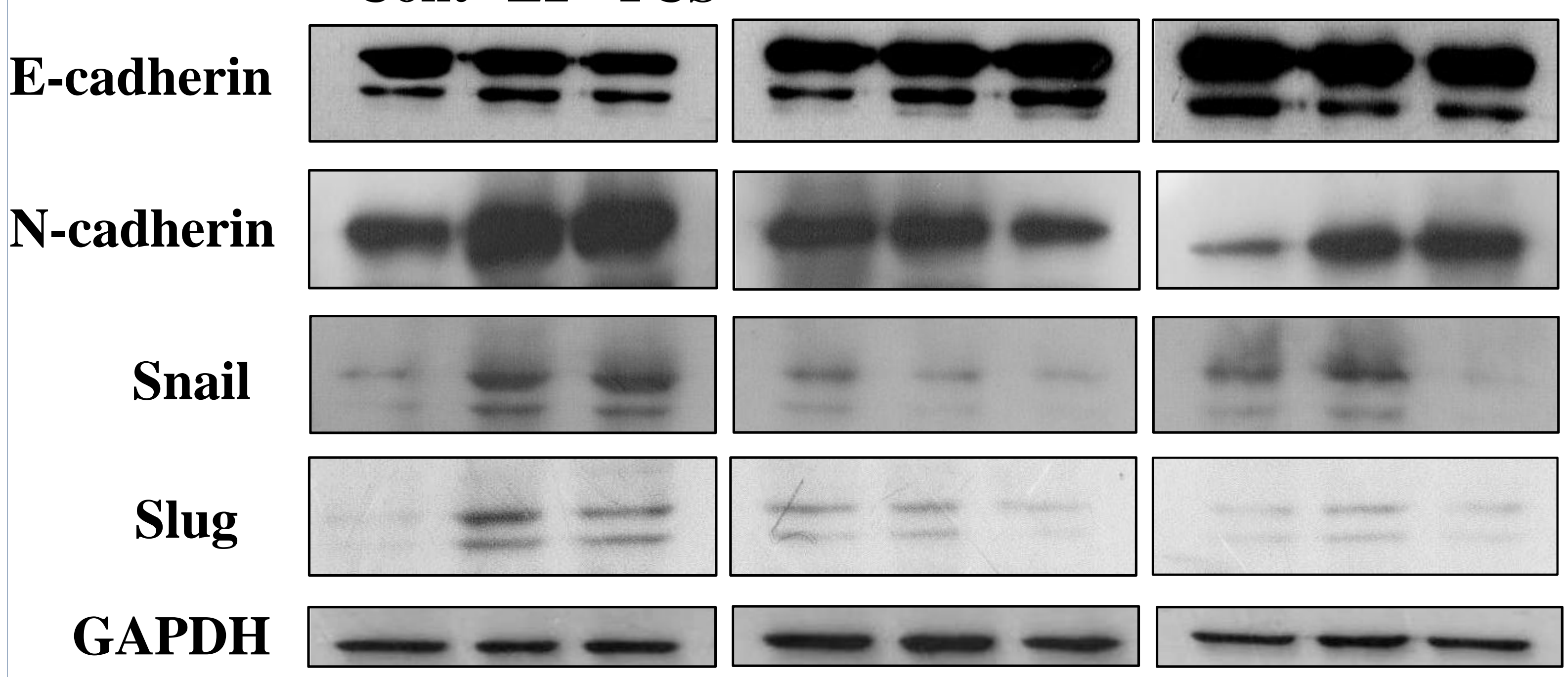

(B)

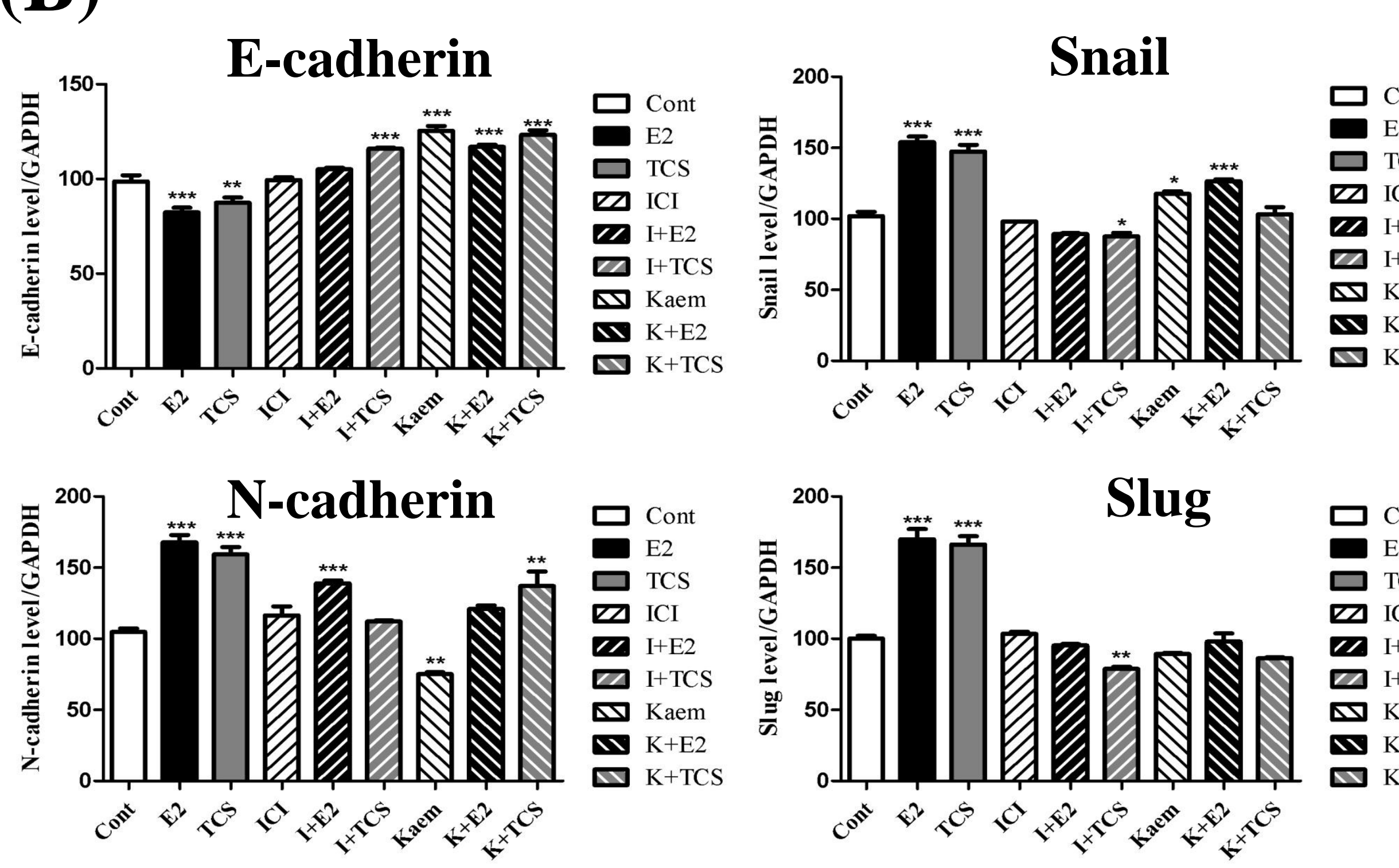

Figure 4. The expression of protein on EMT markers for the effect of E2, TCS, ICI182,720, Kaem in western blot.

The expression of E-cadherin as an epithelial marker was repressed by E2 and TCS treatment compared to the control. In contrast, the expression of N-cadherin Snail and Slug a mesenchymal marker, was significantly incresed by E2 and TCS. ICI182,720 and Kaem up-regulated the expression of E-cadherin and down-regulated in Ncadherin Snail and Slug in western blot (A). Quantification of proteins of E-cadherin, N-cadherin, Snail, Slug and GAPDH were conducted by scanning the densities of bands on a transfer membrane using Gel Doc 2000 (B)
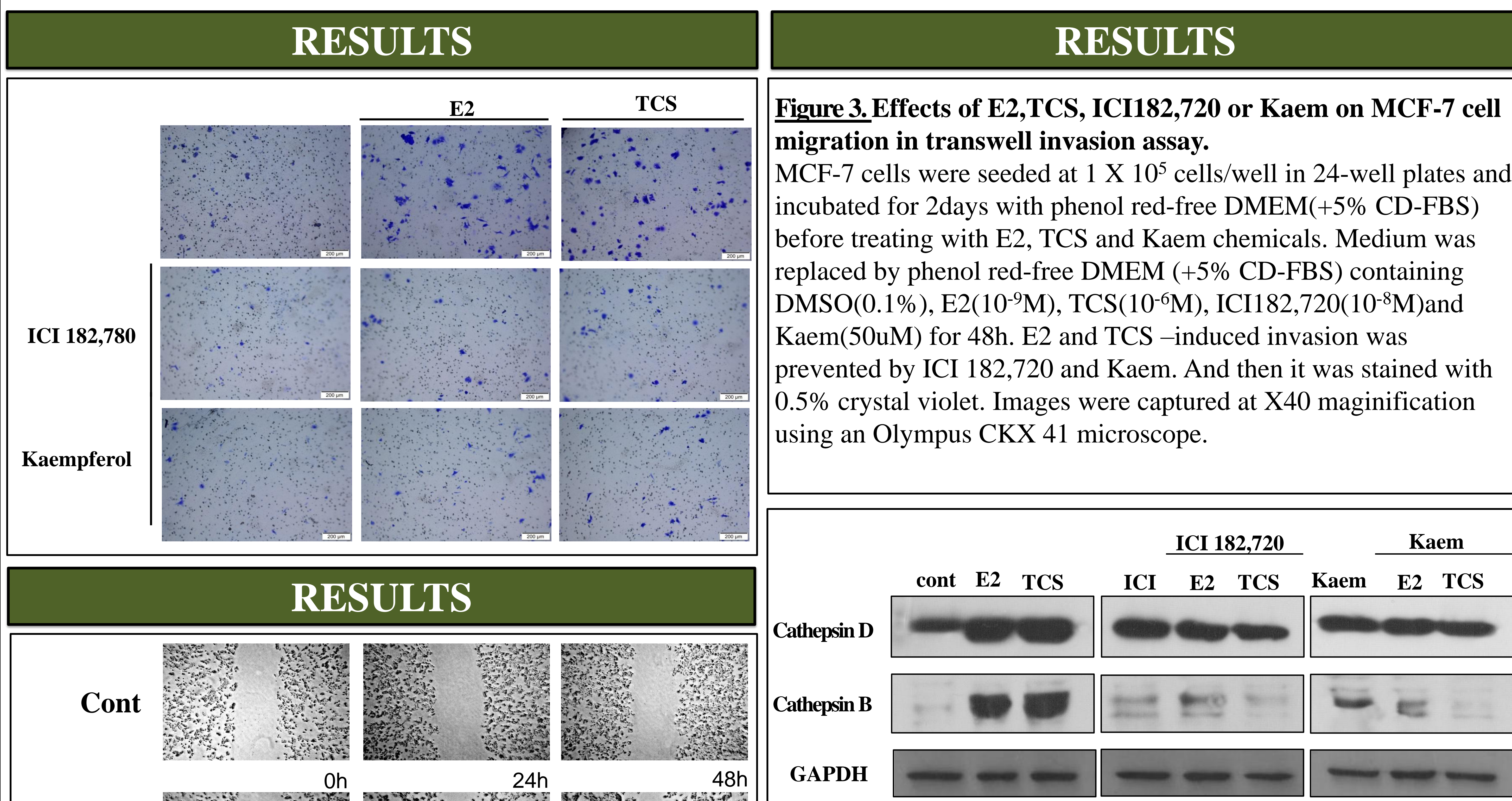

Cont
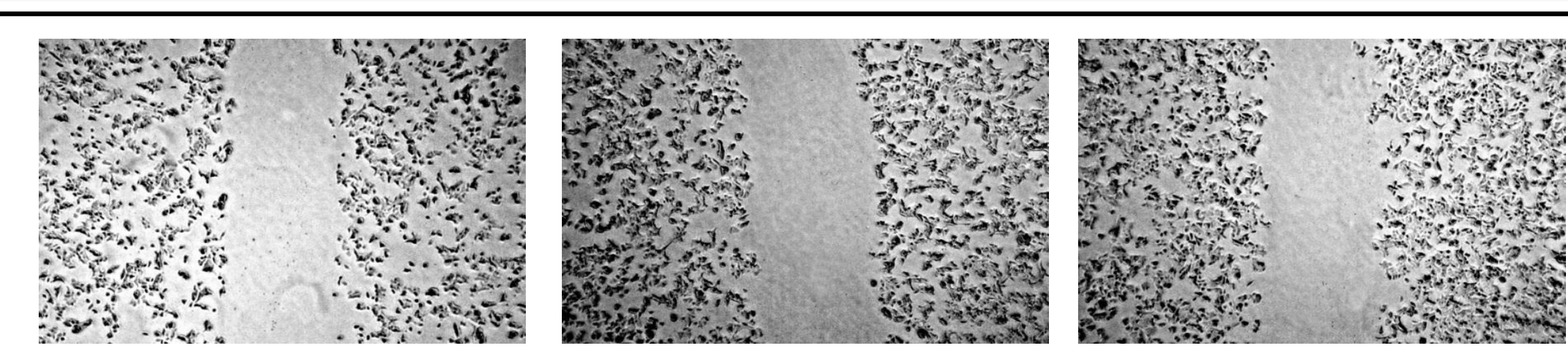

E2
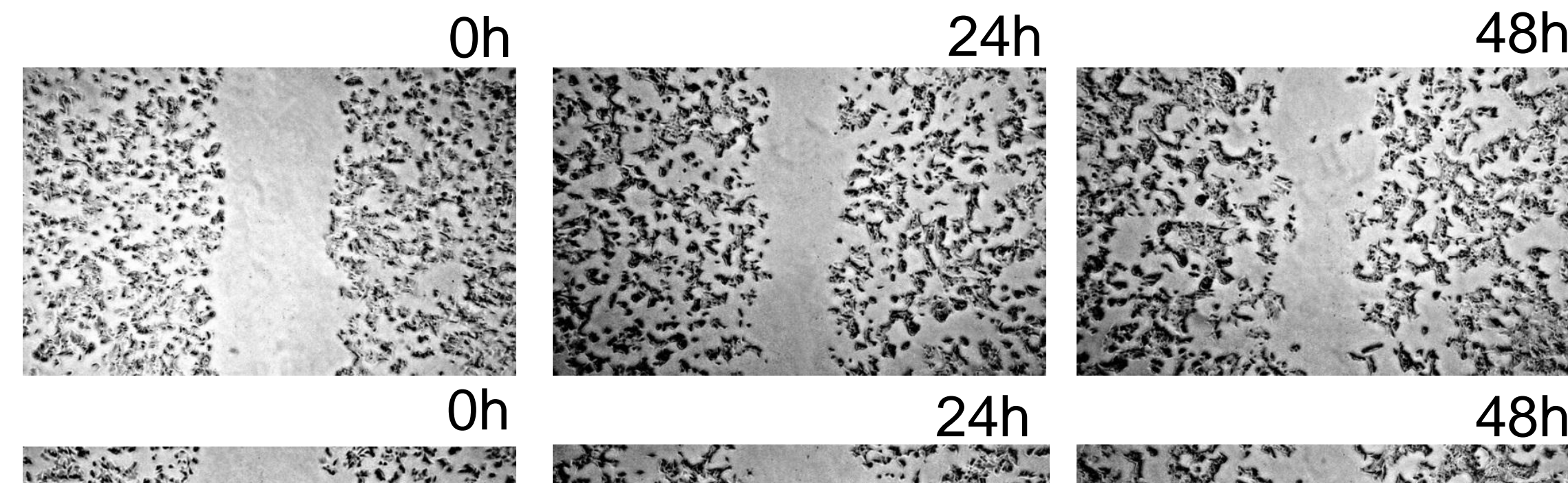

Figure 5. The expression of protein on invasion and metastasis markers for the effect of E2, TCS, ICI182,720, Kaem in western blot The expression of Cathepsin B and D as metastasis markers was increased by E2 and TCS treatment compared to the control. In contrast, the expression of Cathepsin B and D were significantly decreased by ICI 182,720 and Kaem in western blot.

\section{CONCLUSION}

E2+ICI
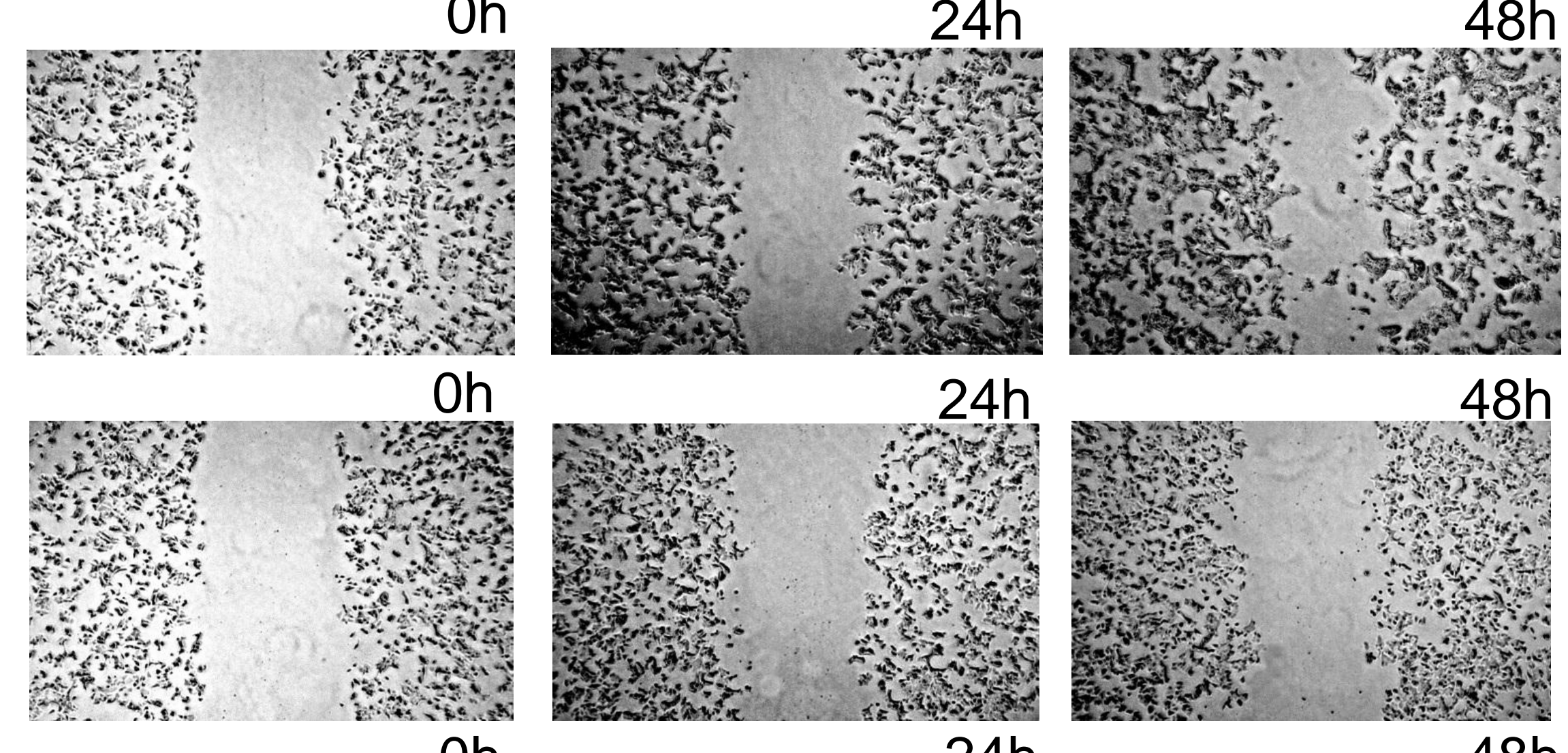

TCS+ICI
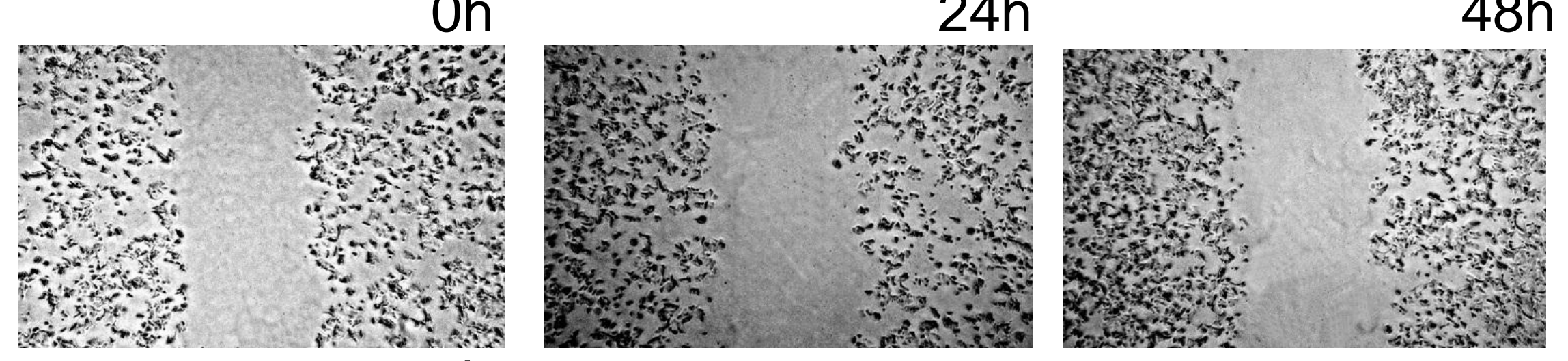

1. E2 and TCS increased proliferation and migration ability of MCF-7 breast cancer cells. And they induced EMT by altering the morphology of MCF-7 cells into the type of mesenchymal cells.

2. Phytoestrogens, Kaempferol, inhibited cell proliferation and migration ability of MCF-7 cells increased by E2 and TCS.

E2+kaem
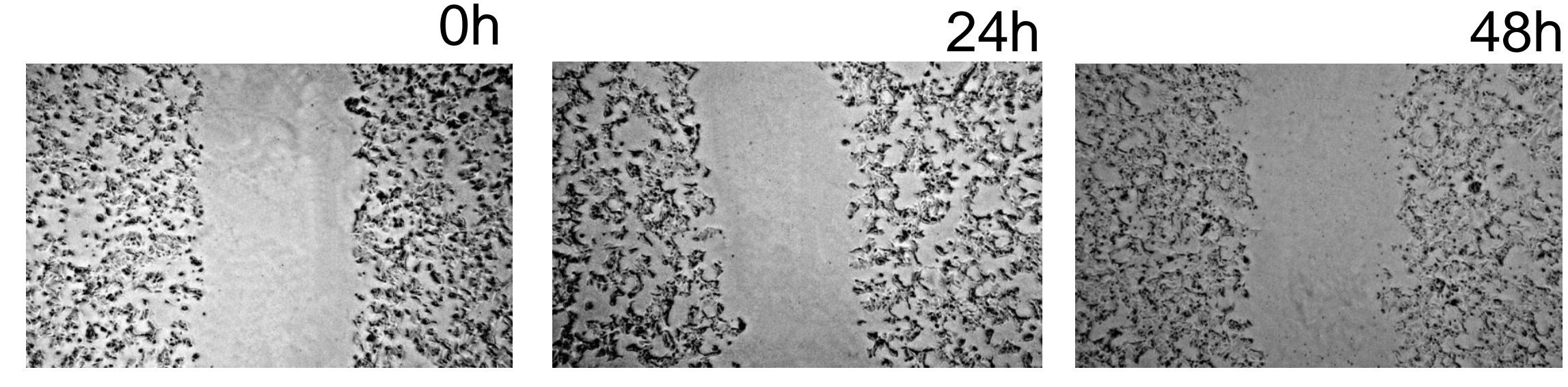

TCS+kaem
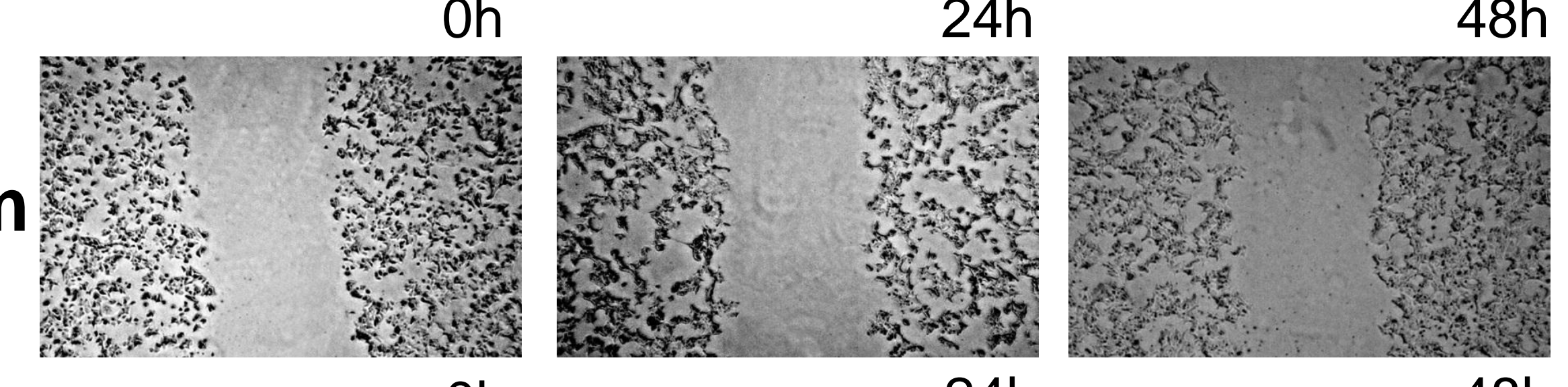

Figure 2. Effects of E2,TCS, ICI182,720 or Kaem on MCF-7 cell migration in a sacatch assay.

MCF-7 cells were seeded at $0.4 \times 10^{6}$ cells/well in 6-well plates and incubated for 2days with phenol red-free DMEM(+5\% CD-FBS) before scratching with a $1 \mathrm{ml}$ pipette tip. Medium was replaced by phenol redfree DMEM $(+5 \%$ CD-FBS $)$ containing $\operatorname{DMSO}(0.1 \%)$, E2 $\left(10^{-9} \mathrm{M}\right)$, $\operatorname{TCS}\left(10^{-6} \mathrm{M}\right)$, ICI1 $82,720\left(10^{-8} \mathrm{M}\right)$ and Kaem(50uM) for $0 \mathrm{~h}, 24 \mathrm{~h}$, and $48 \mathrm{~h}$ E2 and TCS -induced migration was prevented by ICI182,720 and Kaem. Images were captured at X40 maginification using an Olympus CKX 41 microscope. 